\title{
Actualización en biosimilares: una reflexión sobre la reglamentación en Colombia de los medicamentos biológicos y biosimilares
}

\author{
Biosimilars update, a reflection on regulations in Colombia about biological and biosimilar medicines
}

\author{
Juan Raul Castro-Ayarza', César González², Margarita María Velásquez-Loperaª Ángela \\ María Londoño ${ }^{4}$, Zay Beatriz Cárdenas 5 . \\ Miembros del Grupo Colombiano de Psoriasis y Artritis Psoriásica, ColPsor. \\ 1. Médico dermatólogo, especialista en Docencia Universitaria; docente, Universidad Nacional de Colombia, Bogotá, D.C., Colombia \\ 2. Médico dermatólogo, Universidad El Bosque,Bogotá, D.C., Colombia. \\ 3. Médica dermatóloga y doctora en Ciencias Básicas Biomédicas con énfasis en Inmunología; jefe, Sección de Dermatología, Universidad \\ de Antioquia,Centro de Investigaciones Dermatológicas, CIDERM, Medellín, Colombia. \\ 4. Médica dermatóloga, M.Sc. en Epidemiología; docente de posgrado, Universidad CES, Medellín, Colombia. \\ 5. Médica dermatóloga, Universidad Autónoma de San Luis Potosí, San Luis, México; fellow en Cirugía Dermatológica, University of \\ Chicago, Chicago, IL, USA.
}

\section{RESUMEN}

Los medicamentos biológicos para las diferentes enfermedades se encuentran cerca del vencimiento de sus patentes. Las empresas farmacéuticas han empezado a crear medicamentos biosimilares, que se forman a partir de diferentes procesos de producción que los hace medicamentos con similares mecanismos de acción, pero sin ser idénticos a ellos.

La legislación mundial está tomando diferentes posturas para la reglamentación de los medicamentos biosimilares y, en Colombia, se reglamentó recientemente el Decreto 1782 de 2014, con el cual se pretende dar respuesta a esta necesidad.

No obstante, se necesita que como médicos asumamos una posición crítica frente a las diferencias entre los medicamentos biológicos y los biosimilares, conociendo su mecanismo de acción, eficacia, seguridad y capacidad de generar inmunidad, para que la nueva legislación propenda por preservar estos principios, y para evitar la aprobación de medicamentos que no cumplan con los estándares de calidad.

PALABRAS CLAVE: Biosimilares farmacéuticos, medicamentos, legislación en salud.

\section{SUMMARY}

Biologic drugs for various diseases are close to the expiration of their patents. Pharmaceutical companies have began to develop biosimilars, which are formed from different production processes that makes these drugs a similar mechanisms of action, but without being identical to them.

The global law are taking different approaches in order to the regulation of biosimilars and in Colombia, recently regulated by Decree 1782 of 2014, which aims to respond to this need.
Correspondencia:

Juan Raul Castro-Ayarza

Email:

juanraulcastro@yahoo.com

Recibido: 15 de diciembre de 2014 . Aceptado: 10 de febrero de 2015.

Conflictos de interés: Los autores del artículo manifiestan que han participado de forma individual,así: Juan Raul Castro, como asesor de Biopas-Leo Pharma en la línea de psoriasis, y ha sido conferencista de Pfizer; César González, como investigador en protocolos para Laboratorios Abbott, Novartis y Pfizer, y como consultor y conferencista para Laboratorios Wyeth, Schering Plough, Abbott, Abbvie, Pfizer, Merk, Janssen Cilag y Quideca; Margarita Velásquez, como consultora y conferencista para laboratorios Abbott, Abbvie, MSD, Novartis y Pfizer; Ángela Londoño, como investigadora en protocolos para Laboratorios Abbott y Pfizer, consultora y conferencista para Laboratorios Wyeth, Novartis, Abbott, Abbvie, Pfizer y Janssen Cilag; y Zay Beatriz Cárdenas, como miembro de la mesa de conferencistas de Abbvie Latinoamérica. 
However, it is necessary that we as physicians assume a critical position regarding the differences between biologics and biosimilars, knowing its mechanism of action, efficacy, safety and immunogenicity, in order to the new legislation aims to preserve these principles, and to prevent the approval of drugs that do not meet quality standards.

KEY WORDS: Biosimilar pharmaceuticals, drugs, health legislation.

En el mundo existe gran expectativa y preocupación sobre el establecimiento de las políticas de regulación para el uso de medicamentos biológicos en cada país. De igual forma, pacientes y médicos enfrentan la necesidad de que estas políticas estén basadas en el criterio científico y en el beneficio para los pacientes.

Por la naturaleza de estos medicamentos, sus características específicas de producción tienen un impacto en su mecanismo de acción, efectividad y perfil de los efectos adversos, lo que hace que sean difíciles de comparar y reproducir. La sola bioequivalencia no demuestra que sean comparables en términos de eficacia y seguridad en humanos, dados los complejos procesos de producción que involucran sistemas con variabilidad biológica; se considera que, realmente, no es posible hacer dos medicamentos biotecnológicos idénticos. Para evaluar a cabalidad un producto biotecnológico, este debe estar sustentado en ensayos clínicos metodológicamente rigurosos que demuestren su seguridad y eficacia para cada indicación clínica ${ }^{1-3}$.

Los productos biológicos consumen una porción importante del presupuesto nacional para la atención en salud; por lo tanto, la presión financiera para adoptar biosimilares que potencialmente pudieran tener menores costos económicos es alta, no solo en Colombia sino en Latinoamérica y en muchos países del mundo.

Por lo tanto, es conveniente conocer las nuevas tendencias reguladoras que van a tener un impacto en el ejercicio médico. Los cambios en las normativas que pueden tener estas medidas se observarán a mediano y a largo plazo, en la medida en que su implementación ocurra en el ejercicio médico, situación que es conveniente conocer y prever $^{1-3}$.

El conocer el sistema de salud y la normatividad que regula a los productos biológicos y biosimilares, nos permite evaluar el potencial impacto sobre la salud de los individuos y de la comunidad, lo cual no solo es conveniente sino prioritario, para que mantengamos nuestro ejercicio profesional centrado en el beneficio de los pacientes y para que seamos conscientes de nuestra responsabilidad en este proceso. La implementación de las políticas de salud es una construcción colectiva que involucra a entes gubernamentales, asociaciones científicas, médicos, pacientes y a la comunidad en general.

El vencimiento de las patentes de los medicamentos biológicos empleados en dermatología, en las legislaciones de la Food and Drud Administration (FDA) como el etanercept en octubre de 2012, el infliximab en diciembre de 2014 y el próximo vencimiento del adalimumab en diciembre de 2016, estará acompañado de la producción de biosimilares que buscan competir con los originales ${ }^{3,4}$. El vencimiento de las patentes no implica que la compañía farmacéutica del producto innovador revele los detalles de producción, de tal forma que la compañía que desarrolla el biosimilar puede obtener una molécula similar mas no idéntica, lo que da lugar a diferencias entre el innovador y el biosimilar, que pueden cambiar su seguridad y eficacia, como ya ocurrió previamente en los pacientes que presentaron anemia hemolítica por cambios en las diferencias de la solución tampón (buffer) en la producción de la eritropoyetina ${ }^{5}$.

En el mercado, en Colombia, ya existe un medicamento biosimilar comercializado para el etanercept $\left(\right.$ Etanar $\left.^{\circledR}\right)$, el cual fue aprobado bajo una legislación que no tuvo en cuenta estas diferencias entre el medicamento genérico y el biosimilar. En la literatura científica mundial ya se han publicado los primeros estudios clínicos sobre espondilitis anquilosante y artritis reumatoide para el biosimilar de infliximab (Remsina ${ }^{\circledR}$ ) que espera ser comercializado en el país, lo que hace prioritario tener una legislación que permita esta aprobación con la regulación necesaria ${ }^{6,7}$.

La Asamblea Mundial de la Salud expidió la Resolución WHA 67-21 sobre el "acceso a los productos bioterapéuticos, incluidos los productos bioterapéuticos similares y garantía de su calidad, seguridad y eficacia” en la cual se insta a los Estados miembro a establecer las normativas nacionales de evaluación y autorización de medicamentos biológicos. Sin embargo, el Decreto 1782 de 2014 solo toma algunos puntos textuales de dicha resolución, proporcionando así unos parámetros de evaluación y reglamentación con grandes vacíos normativos, con el 


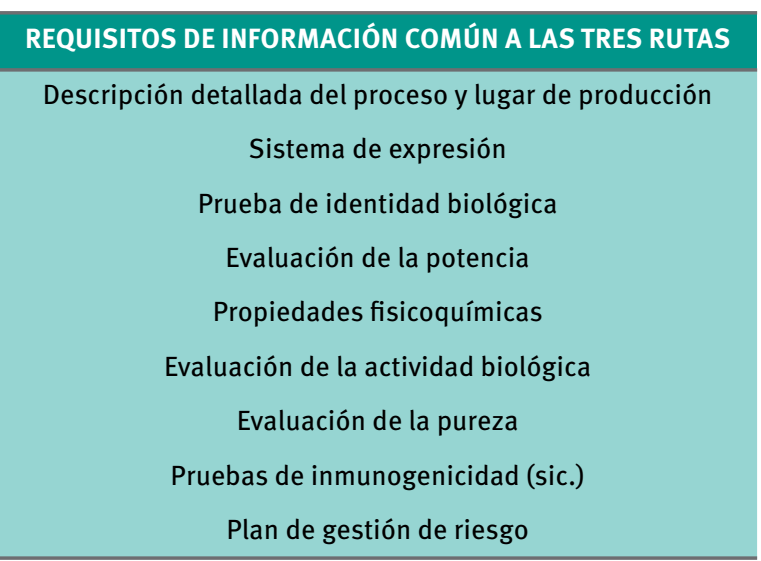

TABLA 1. Decreto 1782 de 2014. Artículo 6. Requisitos de información común a las tres rutas.

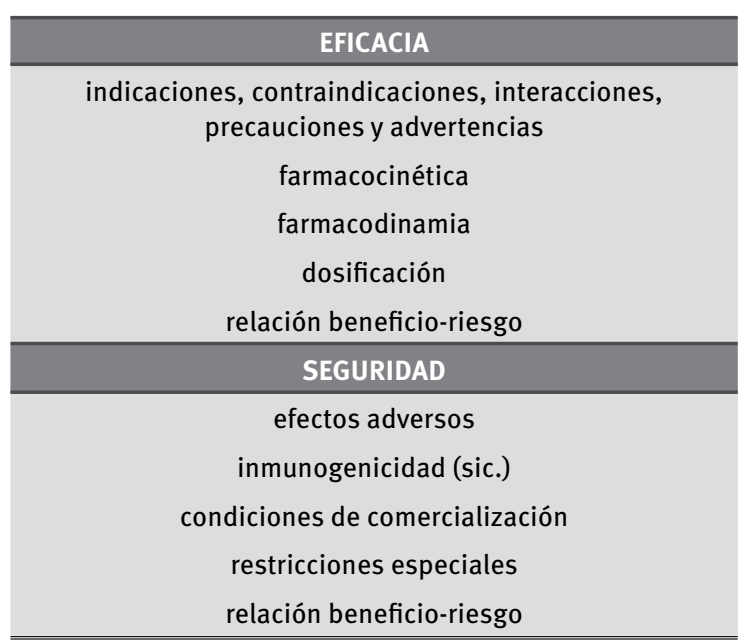

TABLA 2. Decreto 1782 de 2014. Artículo 4. Evaluación farmacológica. riesgo de una posible aprobación de productos biosimilares sin estudios completos y sin fortalecer la reglamentación nacional al respecto ${ }^{8-10}$.

El punto 8 de la sesión 134 del Comité Ejecutivo de la Organización Mundial de la Salud, dice: “[...] Varios países en desarrollo tienen capacidad de producción doméstica para medicinas genéricas y algunos productos médicos relativamente simples. Sin embargo, los sistemas reguladores pueden no estar lo suficientemente desarrollados para regular y controlar los proveedores [...]" ${ }^{11}$. La reflexión apunta a que debe existir una normatividad acorde con estos principios. Al analizar el Decreto 1782 de 2014 , se encuentran vacíos; este aún no es lo suficientemente completo para garantizar que los productos biotecnológicos que se aprueben en el país sean de óptima calidad científica.

Los productos biológicos y biosimilares deben someterse a rigurosas pruebas de control de calidad; la normativa que regula su introducción debe garantizar la eficacia y seguridad de estos productos, razón por la cual muchos países han formulado las directrices para reglamentarlos.

En Colombia, según el Decreto 1782 de 2014, en el contexto de las normas establecidas de la ruta 1-2-3, para ser aprobado, cada medicamento biológico debe cumplir con lo siguiente:

\section{RUTA DEL EXPEDIENTE COMPLETO}

Para la aprobación del medicamento biológico se debe presentar la información común (TABLA 1), el solicitante debe presentar los estudios preclínicos y los ensayos clínicos del medicamento biológico y la evaluación farmacológica (TABLA 2).

\section{RUTA DE LA EQUIVALENCIA}

Se debe presentar la información común (ARTícULo 6, VÉASE LA TABLA 1) y mostrar la similitud en la evaluación farmacológica. Este debe ser aprobado por una sala especializada, según tres criterios: a) el del artículo 27 del Decreto 667 de 1995; b) el de la European Medicine Agency (EMA), la Agencia Nacional de Vigilancia Sanitaria (ANVISA) en Brasil, la Administración Nacional de Medicamento, Alimentos y Tecnología Médica (ANMAT) en Argentina; c) y el de los países de alta vigilancia sanitaria miembros de la Organización para la Cooperación y Desarrollo Económico (OCDE).

\section{RUTA ABREVIADA DE LA POSIBILIDAD DE COMPARACIÓN}

El medicamento puede presentarse con la información de otro país donde haya sido aceptado o puede presentarse con estudios que demuestren que tiene un componente activo muy similar (solo estudios de laboratorio sobre bioequivalencia). La sala especializada tiene la competencia de aprobar o de solicitar información adicional.

La estructura, la conformación y la forma de selección de los miembros de la sala especializada que tiene la competencia para aprobar los productos biotecnoló- 


\begin{tabular}{|c|c|c|}
\hline PARÁMETRO & MEDICAMENTO QUÍMICO & MEDICAMENTO BIOLÓGICO \\
\hline Síntesis & Reproducción de fórmula química & $\begin{array}{l}\text { A partir de la inserción de un gen que } \\
\text { reproduce la molécula en un clon celular }\end{array}$ \\
\hline Tamaño (peso) & $100-1.000 \mathrm{Da}$ & $10.000-300.000 \mathrm{Da}$ \\
\hline Procesos de 'glucosilación' & Nulo & Varios \\
\hline $\begin{array}{l}\text { Capacidad de generar inmunidad } \\
\text { (immunogenicity) }\end{array}$ & Baja & Media a alta \\
\hline $\begin{array}{l}\text { Tiempo de desarrollo de un nuevo } \\
\text { medicamento }\end{array}$ & 7 a 10 años & 10 a 15 años \\
\hline Costos de creación & USD $\$ 500$ a 800 millones & USD\$ 1.200 millones \\
\hline
\end{tabular}

Reimpreso con permiso de: Zelenetz AD, Ahmed I, Braud EL, Cross JD, Davenport-Ennis N, Dickinson BD, Goldberg SE. NCCN Biosimilar White Paper: Regulatory, scientific, and patient safety perspectives. J Natl Compr Canc Netw. 2011;9(Suppl.4):S1-S22. TABLA 3A. Diferencias entre un medicamento químico y un medicamento biológico ${ }^{24}$.

\begin{tabular}{ccc|}
\hline PARÁMETRO & MEDICAMENTO GENÉRICO & MEDICAMENTO BIOSIMILAR \\
\hline Estructura química & Idéntica al producto de referencia & $\begin{array}{c}\text { La secuencia de aminoácidos es la } \\
\text { misma, pero se esperan diferencias } \\
\text { en términos del plegamiento y la } \\
\text { 'glucosilación' de la proteína. }\end{array}$ \\
$\begin{array}{c}\text { Análisis de caracterización } \\
\text { en laboratorio }\end{array}$ & $\begin{array}{c}\text { Sí hay técnicas que identifiquen la } \\
\text { similitud al medicamento original. }\end{array}$ & $\begin{array}{c}\text { No hay técnicas que indiquen que } \\
\text { la molécula es idéntica. Para poder } \\
\text { comparar su seguridad y eficacia, se } \\
\text { requiere realizar estudios clínicos. }\end{array}$ \\
$\begin{array}{c}\text { Tiempo de desarrollo de un } \\
\text { medicamento a partir de una molécula } \\
\text { ya creada }\end{array}$ & 3 años & 6 a años \\
Costo de creación & USD\$1 a 2 millones & USD\$10 a 250 millones \\
\hline
\end{tabular}

Reimpresa con permiso de: Zelenetz AD, Ahmed I, Braud EL, Cross JD, Davenport-Ennis N, Dickinson BD, Goldberg SE. NCCN Biosimilar White Paper: Regulatory, scientific, and patient safety perspectives. J Natl Compr Canc Netw. 2011;9(Suppl.4):S1-S22.

TABLA 3B. Diferencias entre un medicamento genérico y un medicamento biosimilar ${ }^{24}$.

gicos en la ruta de la equivalencia, no están especificadas en el decreto.

En razón de estos tres puntos, se puede decir que el expediente completo y la ruta de la equivalencia son similares a los planteados en otras legislaciones internacionales para la aprobación de medicamentos biosimilares.

La ruta abreviada incluida en la legislación colombiana genera preocupación en su aplicabilidad, pues no representa un verdadero rigor científico que exija a los biosimilares estudios de seguridad y eficacia. En la ruta abreviada, se le entrega a la sala especializada una capacidad de tomar decisiones, que en ningún momento podría reemplazar la necesidad de los estudios preclínicos y clínicos como requisitos de aprobación de biosimilares.
Siendo rigurosos, como la salud de los pacientes lo requiere, no se debería aceptar ningún producto biotecnológico, bien sea innovador o biosimilar, sin estudios clínicos de alta calidad metodológica, realizados específicamente con el medicamento por aprobar para cada indicación clínica.

Preocupa que el Decreto 1782 de 2014 dé lugar a que en Colombia se acepten biosimilares aprobados por nuevas y numerosas agencias reguladoras de menor rigor, tales como la de los países miembros de la OCDE. A esta organización corresponden 34 países, de los cuales existen algunos con niveles de desarrollo, rigor científico y legislaciones poco estrictas en materia del desarrollo de medicamentos biológicos. El decreto abre la posibilidad de 
permitir que en nuestro país se tomen decisiones reguladoras basadas en la experiencia de países cuyos estándares sanitarios son inferiores a los que históricamente ha tenido Colombia, lo que podría afectar negativamente la calidad de los productos biotecnológicos y biológicos que recibirían los colombianos ${ }^{12}$.

La preocupación va mucho más allá de la regulación local; en el ámbito mundial, se hacen alertas sobre los efectos terapéuticos de los medicamentos biológicos, los cuales no se pueden inferir de los estudios de bioequivalencia y, mucho menos, extrapolarlos de entrada a las diferentes indicaciones médicas. Lo anterior se sustenta en que, aunque algunas enfermedades (por ejemplo: psoriasis, enfermedad inflamatoria intestinal, artritis reumatoidea) comparten vías inmunológicas y pueden ser tratadas con el mismo medicamento, su fisiopatología es diferente, lo que puede cambiar los esquemas de manejo, la eficacia y el perfil de los efectos adversos o secunda$\operatorname{rios}^{2,13}$. Es importante entender que un medicamento biológico no es a un biosimilar, lo que es un genérico es a una molécula original ${ }^{1,14,15}$ (TABLA 3, A Y B).

Es probable que un medicamento biosimilar no tenga que seguir todos los pasos del medicamento original para la aprobación en una indicación médica, pero sí debería tener los mismos pasos en términos de ensayos clínicos de seguridad y eficacia. Además, no se debería llegar a la extrapolación directa de un medicamento biosimilaren otras enfermedades que no hayan sido sometidas a estudios clínicos.

Estos fármacos son muchos más complejos en su estructura proteica y son difíciles de replicar; de hecho, no pueden alcanzar la igualdad sino tan solo la similitud, de allí el nombre de biosimilares. Es vital entender que la producción se origina en sistemas con variabilidad biológica, como un gen introducido a nivel celular, que incluyen procesos de 'glucosilación' proteica que cambian las impurezas presentes en la producción, y no en una fórmula a partir de procesos químicos ${ }^{1,2,14}$.

Otro elemento importante que se debe considerar y que no está establecido en todas las regulaciones, es que se puedan intercambiar. En la EMA, la posibilidad de intercambiar entre un medicamento biológico y otro durante el tratamiento de un mismo paciente, se deja a libertad de la legislación de cada estado. La legislación francesa, por ejemplo, ha indicado que, una vez el paciente inicie el tratamiento, el medicamento biológico innovador no puede ser intercambiado por un biosimilar, ni un biosimilar por el producto biológico innovador ${ }^{1,4,10,16}$. En la regulación de la Food and Drug Administración (FDA) de Estados Unidos, hay un papel importante de quien lo prescribe y del paciente frente a la posibilidad de que sean intercambiables y la farmacovigilancia que se legisla en los medicamentos biológicos ${ }^{5}$. En el Decreto 1782 de 2014 no se menciona la posibilidad de que sean intercambiables los medicamentos biológicos durante el tratamiento de un paciente, lo cual genera gran preocupación por parte del médico tratante y del paciente, ante el posible cambio de un medicamento innovador a un biosimilar sin garantía en su eficacia y seguridad. Se hace fundamental que para todos los productos biosimilares se sigan programas serios de farmacovigilancia con capacitación del personal de regulación, información de los efectos secundarios y banco de datos para captura y análisis de los mismos.

Algunos países de Latinoamérica también han establecido criterios para sus reglamentaciones. En el caso de Argentina, la ANMAT cierra la posibilidad de que un medicamento que no haya sido aprobado en un país de referencia, pueda constituirse en una comparación y sólo podrá ser tomado como referente un medicamento biológico autorizado sobre la base de la evaluación de un expediente de registro completo, el cual incluye requisitos de calidad y estudios preclínicos y clínicos. En Argentina no se considera la ruta abreviada, ni la de evidencia global ${ }^{17,18}$.

En el caso de Brasil, la ANVISA considera dos vías para la aprobación de productos biosimilares: el primero, mediante el ejercicio de comparación, al cual le falta claridad conforme a la versión vigente de la guía de la OMS para definir los lineamientos de los estudios de eficacia y de los estudios de seguridad en términos capacidad de generar inmunidad (immunogenicity); y el segundo, mediante desarrollo individual del producto biosimilar que es similar a la introducción del medicamento innovador ${ }^{19}$.

En el caso de México, su normativa está alineada con los estándares de la EMA y la OMS, con algunas mayores exigencias como pruebas de biocomparación (biocomparability) en la población mexicana. La regulación mexicana es más estricta, dado que en años previos ya se presentaron incidentes con moléculas biosimilares ${ }^{20-22}$.

Es importante que los médicos seamos conscientes de la necesidad de estar informados sobre los requisitos que debe cumplir un producto biosimilar para ser aprobado en la legislación colombiana, de la importancia de estar atentos a las pruebas científicas (estudios preclínicos y clínicos de calidad, soportes de usos en las diferentes indicaciones clínicas), de la necesidad de registros de farmacovigilancia con un adecuado reporte de efectos secundarios o adversos y de comprender los riesgos de las sustituciones o intercambios del medicamento sin control $^{3,4}$. El sistema de salud debe estar centrado en la atención y el beneficio del paciente, y todos debemos velar porque la administración de los productos biológicos, 
al igual que cualquier otro medicamento o programa de salud, estén centrados en los pacientes y no en prácticas irregulares que beneficien comercialmente a los involucrados en la atención del paciente.

Un ejemplo de la respuesta que se debería tener ante los vacíos que pueda tener la legislación, es la de Italia, donde diferentes asociaciones médicas (reumatólogos, gastroenterólogos y dermatólogos) se unieron para establecer conceptos de formulación y administración de los medicamentos biosimilares en enfermedades mediadas por mecanismos inmunológicos ${ }^{23}$.

Vemos con preocupación que en el Decreto 1782 de 2014 no hay claridad en algunos puntos y se dejan unos vacíos que podrían no garantizar la bioequivalencia, eficacia y seguridad de los medicamentos biosimilares. La presencia de la ruta abreviada, tal como lo plantea la legislación colombiana, permitiría la aprobación de medicamentos biosimilares sin los estudios clínicos necesarios para demostrar la seguridad y eficacia en las diferentes indicaciones médicas, situación en la cual el dermatólogo debe conocer los diferentes estudios que lo apoyen. Un estricto seguimiento, tanto por el médico como por los pacientes, es necesario para conocer los medicamentos que se están administrando.

La identificación de blancos terapéuticos y el desarrollo de productos biológicos y biosimilares en dermatología son esperanzadores para el manejo de nuestros pacientes. Laboratorios de reconocida trayectoria trabajan tanto en el desarrollo de productos biológicos innovadores como de biosimilares. Las ventajas socioeconómicas de los medicamentos biosimilares parecen estar plenamente comprendidas; sin embargo, es el médico tratante quien debe tomar la decisión de formularlos o no, siendo consciente de la responsabilidad que asume. Se debería negar a utilizar un medicamento biosimilar si existen dudas en la similitud de la molécula, si la seguridad no está claramente establecida, si faltan datos de seguimiento a mediano o a largo plazo, o si el fabricante no genera confianza por falta de datos científicos que lo sustenten. El llamado es a que estos productos deben ser regulados y estudiados apropiadamente antes de su aprobación en Colombia.

\section{REFERENCIAS}

1. Sanclemente G. Lo que debe saber el dermatólogo sobre los medicamentos biológicos y los biosimilares. Rev Asoc Colomb Dermatol. 2011;19:321-9.

2. Puig L. Biosimilares o biosecuelas en Dermatología. Actas Dermosifiliogr. 2010;101:4-6.

3. Torres T, Filipe P, Selores M. Impact of biosimilars in psoriasis treatment. Acta Med Port. 2013;26:646-8.
4. Radtke M, Augustin M. Biosimilars in psoriasis: What can we expect? J Dtsch Dermatol Ges. 2014;12:306-12.

5. Strober B, Armour K, Romiti R, Smith C, Tebbey P, Menter A, et al. Biopharmaceuticals and biosimilars in psoriasis: What the dermatologist needs to know. J Am Acad Dermatol. 2012;66:317-22.

6. Park W, Hrycaj SJ, Jeka S, Kovalenko V, Lysenko G, Miranda P, et al. A randomised, double-blind, multicentre, parallel-group, prospective study comparing the pharmacokinetics, safety, and efficacy of CT-P13 and innovator infliximab in patients with ankylosing spondylitis: The PLANETAS study. Ann Rheum Dis. 2013;72:1605-12.

7. Yoo D, Hrycaj P, Miranda P, Ramiterre E, Piotrowski M, Shevchuk S, et al. A randomised, double-blind, parallel-group study to demonstrate equivalence in efficacy and safety of CT-P13 compared with innovator infliximab when coadministered with methotrexate in patients with active rheumatoid arthritis: The PLANETRA study. Ann Rheum Dis. 2013;72:1613-20.

8. World Health Organization. Access to biotherapeutic products including similar biotherapeutic products and ensuring their quality, safety and efficacy. Item 15.6. Ninth Plenary Meeting, World Health Assembly 67.21; May 24, 2014. Fecha de consulta: 22 de enero de 2015. Disponible en: http://apps.who.int/gb/ ebwha/pdf_files/WHA67/A67_R21-en.pdf.

9. Ministerio de Salud y Protección Social. Decreto 1782 del 18 de septiembre de 2014.Bogotá, D.C., Colombia. Fecha de consulta: 20 de enero de 2015. Disponible en: http://www.minsalud.gov. co/Normatividad_Nuevo/Decreto\%201782\%20de\%202014.pdf.

10. Puig L. Biosimilares en dermatología: infliximab para empezar. Actas Dermosifiliogr. 2013;104:175-80.

11. World Health Organization. Regulatory system strengthening, 134th sesion. A67/32. Secretariat, Executive-Board; 2014. Fecha de consulta: 30 de enero de 2015. Disponible en: http://apps. who.int/gb/ebwha/pdf_files/WHA67/A67_32-en.pdf.

12. González C. Minsalud, 2014. Fecha de consulta: 14 de julio de 2014. Disponible en: http://www.minsalud.gov.co/sites/rid/ Lists/BibliotecaDigital/RIDE/VS/MET/comentarios decreto colpsor.doc.pdf.

13. Carrascosa J. Inmunogenicidad en terapia biológica. Implicaciones en Dermatología. Actas Dermosifiliogr. 2013;104:471-9.

14. Salinas E, Becerra F. Productos biológicos y biosimilares. Diagnóstico (Perú). 2007;46:201-6.

15. Ventola CL. Biosimilars: Part 1: Proposed regulatory criteria for FDA approval. P\&T Journal. 2013;38:270-87.

16. Puig L. Biosimilars in psoriasis 2015: What is next? J Eur Acad Dermatol Venereol. 2014;Nov 11epub.EBSCO. doi 10.1111/ jdv.12843

17. Chiale C. Disposición $N^{\circ}$ 7729/2011. Ministerio de Salud. ANMAT. Administración Nacional de Medicamentos, Alimentos y Tecnología Médica, Secretaría de Políticas. Regulación e Institutos; 21 de noviembre de 2011. Buenos Aires, Argentina. Fecha de consulta: 5 de febrero de 2015. Disponible en: http://ar.vlex. com/vid/disposicion-n-xooao-333633198.

18. Chiale C. Disposición No 7075/2011. Ministerio de Salud. ANMAT. Administración Nacional de Medicamentos, Alimentos y Tecnología Médica., Secretaría de Políticas, Regulación e Institutos; 14 de octubre 2011. Buenos Aires, Argentina. Fecha de consulta: 5 de febrero de 2015. Disponible en: http://www.anmat.gov.ar/ webanmat/retiros/octubre/Disposicion_7075-2011.pdf. 
19. Araujo D. Consideraciones regulatorias sobre productos biológicos en Brasil. Value in Health Regional Issues. 2012;1:254-6.

20. Arriola M. Norma Oficial Mexicana NOM-220-SSA1-2012, instalación y operación de la farmacovigilancia. México, D.F.: Secretaría de Salud, COFEPRIS; 2012.

21. Arriola M. Norma Oficial Mexicana NOM-257-SSA1-2014, en materia de medicamentos biotecnológicos. México, D.F: Secretaría de Salud, COFEPRIS; 2014.

22. Arriola M. Norma Oficial Mexicana NOM-177-SSA1-2013, que establece las pruebas y procedimientos para demostrar que un medicamento es intercambiable. Requisitos a que deben sujetarse los terceros autorizados que realicen las pruebas de intercambiabilidad. Requisitos para realizar los estudios de biocomparabilidad. Requisitos a que deben sujetarse los terceros autorizados, centros de investigación o instituciones hospitalarias que realicen las pruebas de biocomparabilidad. México, D.F.: Secretaría de Salud, COFEPRIS; 2013.
23. Fiorino G, Girolomoni G, Lapadula G, Orlando A, Danese S, Olivieri I, et al. The use of biosimilars in immune-mediated disease: A joint Italian Society of Rheumatology (SIR), Italian Society of Dermatology (SIDeMaST), and Italian Group of Inflammatory Bowel Disease (IG-IBD) position paper. Autoimmun Rev. 2014;13:751-5.

24. Zelenetz AD, Ahmed I, Braud EL, Cross JD, Davenport-Ennis N, Dickinson BD, Goldberg SE. NCCN Biosimilars White Paper: regulatory, scientific, and patient safety perspectives. J Natl Compr Canc Netw. 2011;9(Suppl 4):S1-22. 